\title{
Educational Need Assessment of Infertile Women Admitted to Yazd Reproductive Sciences Institute
}

\author{
Saiideh Norouzi ${ }^{1}$, Zahra Motlagh ${ }^{2 *}$, Nasrin Masoumi ${ }^{1}$, Elahe Tavassoli ${ }^{3}$, Fatemeh Moghaddam ${ }^{1}$
}

\begin{abstract}
Objectives: The purpose of this study was to assess the educational need of infertile women admitted to Yazd Reproductive Sciences Institute.

Materials and Methods: This cross-sectional descriptive-analytical study was conducted in Yazd, Iran. The statistical population included 200 infertile women who were selected through convenience sampling method. The data collection instrument was a researcher-made questionnaire with statistical validity and reliability. Data were collected through interviews and self-reports and then analyzed using SPSS v.16 along with descriptive and analytical statistics.

Results: The mean awareness scores of factors related to infertility, sexual satisfaction and nutritional needs were 4.36, 4.03, and 5.02, respectively. There was a significant relationship between awareness of infertility factors and variables such as level of education, spouse's level of education and place of residence. Moreover, there was a non-significant relationship between awareness of infertility factors and variables such as age, spouse's age, profession, spouse's profession, number of referrals to the institute, and insurance type. There was also a significant relationship between awareness of nutritional needs and profession; whereas a non-significant relationship was seen between the specified awareness and variables such as age, spouse's age, spouse's profession, level of education, spouse's level of education, number of referrals to the institute, place of residence and insurance type.

Conclusion: Since the studied participants exhibited limited awareness of related factors to infertility, it appears that developing a proper training program to raise awareness of infertility followed by its implementation and evaluation is necessary.

Keywords: Needs assessment, Infertility, Yazd, Women
\end{abstract}

\section{Introduction}

The perpetuation of human generations is only possible through pregnancy. To make sure that one's generation is maintained through his offspring is arguably any individual's deepest desire. The willingness to produce children is reinforced by their endearing and appealing nature. Moreover, the desire to ensure survival and leave a precious being behind has been an ever-lasting preoccupation of humankind (1). Available data suggest that $10 \%-15 \%$ of couples suffer from infertility, with more than $90 \%$ of diagnosed cases of infertility successfully treated. Infertility rate is estimated to be $9 \%-22 \%$ among Iranian couples. According to reports by the National Family Health Organization, 3.8\% of women aged 40-44 years are without children (2). Infertile couples often identify infertility as the most stressful event in their life and describe incessant treatment periods as incessant periods of crisis. Infertility has thus attracted the attention of numerous scholars so that, according to Grill, at least 94 quantitative and 26 qualitative studies have been published in this regard since 1986, with many of them revealing greater psychological pressure on infertile women than their male counterparts as well as additional risks due to various reasons (1).

Scholars have diverging viewpoints regarding the im- pact of infertility on couples' relationships: many of them report diminished sexual performance and marital compatibility as a result of infertility; while others maintain that enduring diagnostic and treatment procedures will enhance their relationship and intimacy. Numerous scholars have stressed increased instances of marital arguments and disputes among infertile couples, which could lead to separation in some cases. On the other hand, in most societies infertility is often attributed to women in the early stages of diagnosis and they are the ones who have to shoulder the blame. This, in turn, results in accusations by the husband's family, giving rise to this idea that infertility could be a reasonable ground for the initiation of a divorce or to the husband wedding another woman. On the necessity of finding a solution to the psychological and familial issues of such people, Andrews states: "even if eliminating infertility-related stress is not feasible, attempts should be made to minimize its impacts." Curtailing family disputes and making couples satisfied with their sexual relations and marital life are appropriate ways of mitigating couples' problems (1). To care for themselves, to become healthy and remain healthy, human beings need training and training patients is among the major functions of health-related professions. The philosophy behind training patients is

Received 27 January 2016, Accepted 28 August 2016, Available online 20 September 2016

${ }^{1}$ Abhar School of Nursing, Zanjan University of Medical Sciences, Zanjan, Iran. ${ }^{2}$ Department of Health Education, Faculty of Medical Sciences, Tarbiat Modares University, Tehran, Iran. ${ }^{3}$ Department of Public Health, Faculty of Health, Shahrekord University of Medical Sciences, Shahrekord, Iran.

*Corresponding Author: Zahra Motlagh, Department of Health Education, Faculty of Medical Sciences, Tarbiat Modares University, Tehran, Iran. Tel: +989177036762; Email: motlagh.zahra@yahoo.com 
for them to be able to apply the acquired information and skills to achieve intended purposes. Human beings are eager to identify the kind of lifestyle and manner of conduct that could reduce disease risks and maintain and improve health. Accordingly, helping patients to attain or regain independence in caring for themselves is among the most important of healthcare teams' goals.

In recent decades, a change has been seen in the public attitude with respect to transferring necessary knowledge and meeting educational needs in that members of healthcare teams are expected to offer in-depth information regarding diseases, their complications, method of treatment and self-care to patients and their families in a way that patients are made aware of all relevant aspects to their disease and are able to play a more active self-reliant role in related decisions to one's self. Yet, this is merely possible through interaction and productive communication (3). The outcome of such training is the maintenance and promotion of health in society, with numerous benefits such as reducing disease duration, accelerating the process of patients achieving independence and self-confidence in caring for themselves (3). It should be noted, however, that before providing patients with necessary training, their educational needs have to be specified; otherwise, it will achieve little impact. Educational needs are what any individual should gain an understanding of in order to improve his life condition and promote the society in which he lives (4). Educational need is a gap between an individual's qualifications and competency and higher levels of qualifications that are necessary to reach greater efficacy in the intended organization or society (5). As a general guideline, before the initiation of any training program, the health needs and concerns of individuals have to be specified and evaluated first. To achieve this, it is necessary to develop an understanding of trainees' level of knowledge, and health attitude and behavior (4-6). This study was thus devised and carried out with the purpose of assess the educational needs of infertile women admitted to Yazd Reproductive Sciences Institute.

\section{Materials and Methods}

This cross-sectional descriptive-analytical study was conducted in January-February 2015 in Yazd, Iran. The statistical population included the infertile women admitted to Yazd Reproductive Sciences Institute who were selected through convenience sampling method. 200 individuals were selected as the sample size based on previous studies. Inclusion criteria included women who were unable to conceive for more than one year and the completion of informed consent forms. Questionnaires were distributed to participants after explaining research objectives in a comprehensive way and obtaining their consent and willingness to take part in this study. They were also assured that the collected data will remain confidential and ultimate care will be exercised to prevent any physical or psychological harm to the target group.

The data collection instrument was a researcher-made questionnaire with statistical validity and reliability. To design the questionnaire, a qualitative study was initially conducted: interviews were conducted with 20 women admitted to the infertility center on a random basis and the questionnaire was developed following the analysis of results. In addition to demographic items, this questionnaire also contained the following items under 4 sections: 1) Awareness of related factors to infertility (20 items), 2) awareness of nutritional needs (6 items), 3) sexual satisfaction (5 items), and 4) general items related to infertility. Each correct answer from sections 2 and 3 was given 1 point, while wrong and "I do not know" answers were given zero points. Since the aim was to determine awareness levels, the "I do not know" answers were also assigned zero points. For section 4, each affirmative answer was given 1 point and negative and "I do not know" answers were given zero points.

The questionnaire's content and face validity was determined based on the viewpoints of 5 experts in the field of health and infertility education, and its construct validity was obtained through factor analysis. A qualitative content analysis was first conducted to design the questionnaire. Its categories and themes were extracted based on which the questionnaire was developed. Then, alpha was calculated for questionnaire. Alpha coefficient was obtained following: 1) Awareness of related factors to infertility ( $\alpha=80), 2)$ swareness of nutritional needs $(\alpha=81)$ and 3) sexual satisfaction $(\alpha=83)$ and, 4$)$ general items related to infertility $(\alpha=85)$.

Data were collected through interviews and self-reports and analyzed using SPSS version 16. Descriptive statistics were performed to discover means \pm standard deviation (SD) for incessant variables and percentage (\%) for definite variables, correspondingly, Mann-Whitney and Kruskal-Wallis tests were also performed. For all tests, the meaning level of a ( $P$ value) was considered as 0.05 .

\section{Results}

The mean age of participants and participants' spouses was $28 \pm 5.22$ and $32.26 \pm 5.64$ years, respectively. The frequency distribution of demographic variables is presented in Table 1.

$86.6 \%$ (207 individuals) lowered their cost of living to be able to treat infertility and $81.1 \%$ (193 individuals) were confident of completing their treatment procedure. $80.3 \%$ (191 individuals) were certain that their infertility would be successfully treated. $41.6 \%$ (97 individuals) of the participants regarded infertility as a mere medical condition, $50.9 \%$ (118 individuals) a social issue, 68\% (157 individuals) an economic problem and $81.2 \%$ (190 individuals) an emotional challenge.

$65.7 \%$ (151 individuals) were aware of the cause behind their or their spouse's infertility. 48.8\% (93 individuals) stated that the cause of infertility lied in them and $28.9 \%$ (66 individuals) stated that it lied in their spouse. $69.6 \%$ (160 individuals) and $61.1 \%$ (140 individuals) were aware of the treatment type and received diagnostic procedures. Medication was prescribed for $75.4 \%$ (169 individuals), with $75.1 \%$ (160 individuals) knowing how and when to 
Table 1. The Frequency Distribution of Demographic Variables of Women Admitted to Yazd Reproductive Sciences Institute

\begin{tabular}{|c|c|c|}
\hline Variables & Number & $\%$ \\
\hline \multicolumn{3}{|l|}{ Job } \\
\hline Housewife & 184 & 54.7 \\
\hline Have a job & 63 & 55.2 \\
\hline \multicolumn{3}{|l|}{ Occupation of husband } \\
\hline Employee & 93 & 87.3 \\
\hline Working & 41 & 16.7 \\
\hline Free & 109 & 44.3 \\
\hline Jobless & 3 & 1.2 \\
\hline \multicolumn{3}{|l|}{ Address } \\
\hline Yazd & 88 & 34.9 \\
\hline Out of Yazd & 164 & 65.1 \\
\hline \multicolumn{3}{|c|}{ Referring to the history center } \\
\hline Yes & 137 & 55.5 \\
\hline No & 110 & 44.5 \\
\hline \multicolumn{3}{|l|}{ Wife's level of education } \\
\hline Degree cycle & 54 & 22 \\
\hline Diploma & 93 & 37.8 \\
\hline License & 99 & 40.2 \\
\hline \multicolumn{3}{|l|}{ Husband's education level } \\
\hline Degree cycle & 51 & 20.9 \\
\hline Diploma & 104 & 42.6 \\
\hline License & 89 & 36.5 \\
\hline \multicolumn{3}{|c|}{ No. of times referring to infertility centers } \\
\hline Once & 65 & 47.1 \\
\hline Twice & 28 & 20.3 \\
\hline Three times and more & 45 & 32.6 \\
\hline \multicolumn{3}{|l|}{ Insurance } \\
\hline Social security & 161 & 69.1 \\
\hline Health Service & 44 & 18.9 \\
\hline Other & 28 & 12 \\
\hline
\end{tabular}

take them.

Major concerns of infertile couples from the participants' perspective, respectively included: lack of health insurance coverage with $36.4 \%$ (76 individuals), concern about negative reaction by other family members and the society to infertility with $24.9 \%$ (52 individuals), the ambiguous nature of their rights in the national health system with $20.6 \%$ (43 individuals) and of supporting organizations with $6.7 \%$ (14 individuals). $84.3 \%$ of the participants were willing to receive detailed information of their treatment process from the medical team. The frequency distribution of related items to infertility awareness, sexual satisfaction and nutritional needs is given in Tables 2-4.

Results show that 189 individuals (75\%) were content with their ability to reach orgasm during sexual intercourse, with 63 individuals (25\%) replying with negative or "I do not know" answers. 195 individuals (77.7\%) were satisfied of their arousal level during sexual performance, with 56 individuals $(22.3 \%)$ choosing negative or "I do not know" answers. 219 individuals (87.3\%) were satisfied of the emotional intimacy between them and their spouses during sexual intercourse and 32 (12.7\%) answered with "no" or "I do not know." A total of 208 individuals (82.9\%) were content of their sexual relations with their spouse and $43(17.1 \%)$ chose "no" or "I do not know." A total of 205 individuals (83.3\%) were generally content of their sex life, while 41 individuals (16.7\%) opted for "no" or "I do not know" answers.

The feasible region, observed region, mean score, and standard deviation of awareness of infertility factors, sexual satisfaction, and awareness of nutritional needs are presented in Table 4.

Results from the Mann-Whitney test revealed that there was a significant relationship between awareness of infertility factors and place of residence $(P=0.018)$ and a non-significant relationship between the former and history of referral to the treatment center $(P=0.463)$. The Kruskal-Wallis test results revealed that there was a significant relationship between awareness of infertility factors and level of education $(P=0.000)$ and spouse's level of education $(P=0.008)$; whereas no significant relationship was seen between awareness of infertility factors and age $(P=0.192)$, spouse's age $(P=0.313)$, profession $(P=0.327)$, spouse's profession $(P=0.160)$, number of referrals to the institute $(P=0.696)$ and insurance type $(P=0.620)$.

Results from the Mann-Whitney test pointed to no significant relationship between sexual satisfaction and place of residence $(P=0.344)$ and history of referral to the institute $(P=0.794)$. The Kruskal-Wallis test results demonstrated a significant relationship between sexual satisfaction and education level $(P=0.036)$ and a non-significant relationship between the former and such variables as age $(P=0.607)$, spouse's age $(P=0.221)$, profession $(P=0.411)$, spouse's profession $(P=0.564)$, spouse's level of education $(P=0.077)$, number of referrals to the institute $(P=0.239)$ and insurance type $(\mathrm{p}=0.802)$.

Results from the Mann-Whitney test showed no significant relationship between awareness of nutritional needs and place of residence $(P=0.246)$ and history of referral to the institute $(P=0.912)$. The Kruskal-Wallis test results demonstrated a significant relationship between awareness of nutritional needs and profession $(P=0.002)$ and a non-significant relationship between the former and such variables as age $(P=0.335)$, spouse's age $(P=0.665)$, spouse's profession $(P=0.281)$, level of education $(P=0.149)$, spouse's level of education $(P=0.085)$, number of referrals to the institute $(P=0.128)$ and insurance type $(P=0.854)$.

\section{Discussion}

The mean infertility awareness score was $11 \pm 4.36$, pointing to a poor awareness level on the part of participants. In the study by Dyer on 150 infertile women, close to half of them expressed a lack of knowledge of infertility causes (7). In the study by Quach and Librach, high school students' awareness of infertility prevention measures was limited (8). Another study by Ali et al on 247 adults pointed to evident insufficient awareness levels. In a global-scale study on 17500 individuals, it was noticed that awareness of infertility and the biology of reproduction 
Table 2. The Frequency Distribution of Related Items to Infertility Awareness

\begin{tabular}{|c|c|c|c|c|}
\hline \multirow{2}{*}{ Question } & \multicolumn{2}{|c|}{ Right } & \multicolumn{2}{|c|}{ Wrong and I do not know } \\
\hline & No. & $\%$ & No. & $\%$ \\
\hline What is aging effect on the fertility of men or women? & 194 & 77.6 & 56 & 22.4 \\
\hline What is the effect of history of damage to the testicles on male fertility? & 182 & 72.8 & 68 & 27.2 \\
\hline What is the effect of surgery on male fertility? & 53 & 21.3 & 196 & 78.7 \\
\hline What is the effect of history of mumps on male fertility? & 92 & 36.8 & 158 & 63.2 \\
\hline What is the effect of febrile illness on male fertility? & 84 & 33.6 & 166 & 66.4 \\
\hline What is the effect of very hot baths on male fertility? & 86 & 34.4 & 164 & 65.6 \\
\hline Tight clothing: What is the effect on male fertility? & 118 & 46.8 & 134 & 53.2 \\
\hline Sitting jobs or exposed to heat, such as driving and bakery: What is the effect on male fertility? & 104 & 41.6 & 146 & 58.4 \\
\hline Severe allergic reaction (allergic): What is the effect on male or female fertility? & 66 & 26.4 & 184 & 73.6 \\
\hline Environmental or industrial-rays: What is the effect on male or female fertility? & 158 & 63.7 & 90 & 36.3 \\
\hline What is the effect of excessive alcohol consumption on male fertility? & 171 & 68.1 & 80 & 31.9 \\
\hline What is the impact of drug use on male fertility? & 172 & 68.5 & 79 & 31.5 \\
\hline What is the effect of smoking on male fertility? & 150 & 60.7 & 97 & 39.3 \\
\hline $\begin{array}{l}\text { Sperm count, percent of sperm animated shapes, size and shape of sperm quality and male } \\
\text { fertility have impact on? }\end{array}$ & 51 & 20.7 & 195 & 79.3 \\
\hline Thyroid disease in women, what impact they will have on fertility? & 169 & 68.1 & 79 & 31.9 \\
\hline $\begin{array}{l}\text { If you have a regular menstrual cycle and premenstrual symptoms associated: what effect it has } \\
\text { on women's fertility? }\end{array}$ & 168 & 68.8 & 77 & 31.4 \\
\hline What impact does ovulation disorder have on women's reproduction? & 189 & 77.1 & 56 & 22.9 \\
\hline Damage or blockage of the fallopian tubes: impact on women's fertility? & 196 & 80.3 & 48 & 19.7 \\
\hline Pelvic infections: What impact it has on women's reproductive? & 181 & 73.9 & 64 & 26.1 \\
\hline Adhesions around the fallopian tubes and ovaries: Impact on women's fertility? & 189 & 77.5 & 55 & 22.5 \\
\hline
\end{tabular}

Table 3. The Frequency Distribution of Related Items to Awareness of Nutritional Needs

\begin{tabular}{|c|c|c|c|c|}
\hline \multirow{2}{*}{ Question } & \multicolumn{2}{|c|}{ Right } & \multicolumn{2}{|c|}{ Wrong and I Do not Know } \\
\hline & No. & $\%$ & No. & $\%$ \\
\hline Is the daily consumption of 3 cups of milk for protein and calcium necessary? & 224 & 89.6 & 26 & 10.4 \\
\hline Is daily intake of lean meat, poultry, fish and eggs at a rate of 120 to $180 \mathrm{~g}$ for protein necessary? & 209 & 83.3 & 42 & 16.7 \\
\hline Is the consumption of raw and cooked vegetables and grains at a rate of 3 to 5 units necessary? & 216 & 86.4 & 34 & 13.6 \\
\hline Is the daily consumption of fruits by 2 to 4 units is required? & 219 & 87.3 & 32 & 12.7 \\
\hline Is the daily intake of whole grain breads, cereals by more than 7 units necessary? & 162 & 64.5 & 89 & 35.5 \\
\hline Do not consume 6 to 8 glasses of water a day: Is it healthy? & 234 & 93.2 & 17 & 6.8 \\
\hline
\end{tabular}

was poor throughout the world (9), which corresponds to the present study. In the study by Bunting and Boivin on 149 students pursuing bachelor's or master's degrees, $90.7 \%$ of them accurately identified infertility risk factors (10), which does not correspond to our study. Lower awareness levels in this study can be attributed to lower education levels on the part of participants compared to those of the specified study.

In this study $77.6 \%$ of participants maintained that infertility is diminished by age in both men and women. In the study by Daniluk et al on 3343 childless women, $90.3 \%$ of them were aware of this fact (11). In the study by Quach and Librach infertility is influenced by women's age according to $84 \%$ of female high school students (8). On another study by Bretherick et al on 360 bachelor's students, $70.3 \%$ of women recognized that fertility is significantly diminished before menopause and $45.5 \%$ regarded age as the most powerful infertility risk factor in women (12) which corresponds to the findings of this study.

According to $60.7 \%$ of participants in this study, smok- ing cigarette diminishes fertility in men. In the study by Quach and Librach, 66\% of high school students identified cigarette smoking as a factor influencing fertility (8), which matches the findings of this study. In the study by Roth and Taylor on 388 female hospital staff, $22.3 \%$ established a correlation between smoking cigarette and infertility (13). In the study by Ali et al, $76 \%$ of adults did not view smoking as a cause of infertility (9) which does not correspond to our study.

In the present study, $68.1 \%$ of participants viewed excessive consumption of alcohol as being related to diminished infertility in men. In the study by Quach and Librach 66\% of high school students identified excessive consumption of alcohol as a detrimental factor (8). This is in line with the findings of our study. In this study, awareness of infertility was not correlated to age, which is corroborated in the study of Bunting and Boivin (10).

In the present study, $73.9 \%$ of participants stated that pelvic infection diminishes female infertility. In the study by Ali et al, 705 of participants identified vaginal infection 
Table 4. The Feasible Region, Observed Region, Mean Score, and Standard Deviation of Awareness of Infertility Factors, Sexual Satisfaction, and Awareness of Nutritional Needs

\begin{tabular}{lcccc}
\hline Variable & Feasible Region & Observed Region & Mean Score & SD \\
\hline Awareness of infertility factors & $0-20$ & $0-19$ & 4.36 & 4.03 \\
Sexual satisfaction & $0-5$ & $0-5$ & 11 & 5.02 \\
Awareness of nutritional needs & $0-6$ & $0-6$ & 1.30 & \\
\hline
\end{tabular}

Abbreviation: SD, Standard Deviation.

as a cause of infertility (9). In the study by Daniluk et al, $82.3 \%$ of childless women were aware that sexually transmitted diseases enormously increase the risk of secondary infertility (11). In the study by Quach and Librach, 57.5\% of participants were aware that sexually transmitted diseases could lead to infertility (8). A number of sexually transmitted diseases can lead to pelvic infection, which in turn results in infertility. These findings correspond to the findings of the present study. In the specified study, $81 \%$ of students did not know what pelvic infection was (8) which does not correspond to our study. This could be accounted for by the marital status and greater experience of the participants of this study compared to those of the specified research, i.e., single students.

According to $68.6 \%$ of the participants of this study, regular menstrual cycle increases fertility. In the study by Ali et al, more than $70 \%$ of participants identified irregular menstrual cycle as an infertility factor (9). In the study by Dyer et al, the majority of women regarded irregular menstrual cycles on their part as a reason for voluntary childlessness (14), which corresponds to this study.

$80.3 \%$ of the participants of this study identified damaged or obstructed fallopian tube as a factor diminishing infertility in women, which was corroborated in the study by Ali et al, in more than $70 \%$ of the studied subjects (9). In the study by Dyer et al, the majority of women attributed their voluntary childlessness to fallopian tube obstruction (14), which corresponds to the findings of this study.

In the present study, $83.6 \%$ of participants stated that adopting children is of spiritual merit. In the study by Ali et al, adults had a completely positive attitude to adoption, with 92\% regarding it an acceptable alternative for infertile couples (9), which corresponds to this study.

Sexual satisfaction levels were favorable in this study. In the studies conducted by Bahrami et al and Nourani et al, infertile women reported average sexual satisfaction $(15,16)$, which corresponds to the findings of this study. In the study by Pazandeh et al, sexual problem was seen as a possibility in $100 \%$ of infertile women, meaning that all participants suffered from sexual dissatisfaction (17). This is in line with the results of this study.

A significant relationship was noticed between sexual satisfaction and level of education in this study, which was verified in the study by Bakhshayesh and Mortazavi (18). In the study by Seif et al, women's level of education is a strong predictor of their satisfaction of future life (19). Sexual satisfaction was not found to be related to age or profession, which was similar to the findings of Bahrami et al (15). No significant relationship was also found between sexual satisfaction and age in the study by Rah- mani et al (20), which is in line with the findings of this research. In the study by Faal Kalkhoran et al, a significant relationship was noticed between sexual satisfaction and age in the infertile group (21), which is not consistent with our findings.

In this study, $14.9 \%$ of infertile women had resorted to sports clubs to distract themselves from their problem. Mohammadi Hosseini et al found that infertile women had never made use of relaxation techniques (22). In the study by Latifnejad Roudsari et al, $80.8 \%$ of fertile women took part in regular sports activities (23), which does not correspond with our study.

In this study, $80.3 \%$ of infertile women had faith that their condition would be successfully treated. In the study by Latifnejad Roudsari et al, 56.9\% of participants were completely optimistic about their successful treatment (23).

According to $85.6 \%$ of infertile women in this study, religious belief plays a positive role in helping them cope with their problem. In the study by Mohammadi Hosseini et al, the majority of participants consistently used three emotion-oriented strategies in praying, trusting in God, and anxiety and weeping (22).

\section{Conclusion}

Since the studied participants exhibited limited awareness of related factors to infertility, it appears that developing a proper training program to raise awareness of infertility followed by it implementation and evaluation is necessary.

\section{Ethical Issues}

The study was reviewed and approved by ethical committees of Yazd Reproductive Sciences Institute. The process of sampling and examination began after explaining the project to the women. Participation in the study was voluntary. Written informed consent was obtained from women.

\section{Conflict of Interests}

We declare that there is no conflict of interests.

\section{Financial Support \\ None}

\section{Acknowledgments}

The researchers would like to thank Reproductive Sciences Institute and all women who participated in the research.

\section{Reference}

1. Mollaiy Nejad M, Jaaferpour M, Jahanfar Sh, Jamshidi R. 
Infertility related stress and marital life in Iranian infertile women who referred to Isfahan infertility treatment clinic. J Reprod Infertil. 2001;2(1):26-39.

2. Khodakarami N, Seddiq S, Hashemi S, Hamdieh M, Taheripanah R. The disregarded rights of infertility: a phenomenological study. Iranian J Med Ethics History Med. 2009;2(3):39-50.

3. Mohammad Pour A, Dehgan Naieri N. The survey of the patient educational need on discharge phase in Gonabad health and treatment centers. Horizon Med Sci. 2007;4(12):34-40.

4. Ghobadi SS, Negharandeh R. Determine the training needs of women referred to the health center's number one city of Zanjan on some family planning methods. ZUMS J. 2001;9(34):41-46.

5. Dattijo L, Andreadis N, Aminu B, Umar N, Black K. Knowledge of infertility among infertile women in Bauchi, Northern Nigeria. Int J Women Health Reprod Sci 2016;3(4):102-109. doi:10.15296/ijwhr.2016.25.

6. Andalib M, Hassanzadeh R, Bakhshi FM. Evaluation of the effect of corrected nutrition on reduction of stress in gestational diabetes. Int J Women Health Reprod Sci. 2016;3(4):114-8. doi:10.15296/ijwhr.2016.27.

7. Dyer SJ. Infertility-related reproductive health knowledge and help-seeking behaviour in African countries. ESHRE Monographs. 2008;(1):29-33. doi:10.1093/humrep/den148.

8. Quach S, Librach C. Infertility knowledge and attitudes in urban high school students. Fertil Steril. 2008;90(6):20992106. doi:10.1016/j.fertnstert.2007.10.024.

9. Ali S, Sophie R, Imam AM, Khan FI, Ali SF, Shaikh $\mathrm{A}$, et al. Knowledge, perceptions and myths regarding infertility among selected adult population in Pakistan: a cross-sectional study. BMC Public Health. 2011;11(1):1. doi:10.1186/1471-2458-11-760.

10. Bunting L, Boivin J. Knowledge about infertility risk factors, fertility myths and illusory benefits of healthy habits in young people. Hum Reprod. 2008;23(8):1858-1864. doi: 10.1093/humrep/den168.

11. Daniluk JC, Koert E, Cheung A. Childless women's knowledge of fertility and assisted human reproduction: identifying the gaps. Fertil Steril. 2012;97(2):420-426. doi:10.1016/j.fertnstert.2011.11.046.

12. Bretherick KL, Fairbrother N, Avila L, Harbord SH,
Robinson WP. Fertility and aging: do reproductive-aged Canadian women know what they need to know? Fertil Steril. 2010;93(7):2162-8. doi:10.1016/j.fertnstert.2009.01.064.

13. Roth LK, Taylor HS. Risks of smoking to reproductive health: assessment of women's knowledge. Am J Obstet Gynecol 2001;184(5):934-9. doi:10.1067/mob.2001.112103.

14. Dyer SJ, Abrahams N, Hoffman M, van der Spuy ZM. Infertility in South Africa: women's reproductive health knowledge and treatment-seeking behaviour for involuntary childlessness. Hum Reprod. 2002;17(6):16571662. doi:10.1093/humrep/17.6.1657.

15. Bahrami N, Sattarzadeh N, Ranjbar Koochaksariie F, Pezeshki MZ. Comparing depression and sexual satisfaction in fertile and infertile couples. J Reprod Infertil. 2007;8(1):52-59.

16. Nourani Sh, Jonaidi E, Shakeri MT, Mokhber N. Sexual satisfaction in fertile and infertile women attending state clinics in Mashad. J Reprod Infertil. 2010;10(4):269-277.

17. Pazandeh F, Sharghi Somea N, Karami Nouri R, Alavi Majd H. Investigate the relationship between well-being and happiness of marriage infertility. Pajoohandeh J. 2005;9(42):355-360.

18. Bakhshayesh AR, Mortazavi M. The relationship between sexual satisfaction, mental health and marital satisfaction in couples. J Appl Psychol. 2010;3(4):73-85.

19. Seif D, Alborzi SH, Alborzi S. Effect of some affective and demographic variables on life satisfaction of infertile women. J Reprod Infertil. 2001;2(4):66-74.

20. Rahmani A, Sadeghi N, Jafarpoor M, Merghati-Khoei EA. The relation of sexual satisfaction and demographic factors. Iran J Nursing. 2010;23(66):14-22.

21. Faal Kalkhoran L, Bahrami H, Farrokhi NA, Zeraati H, Tarahhomo M. Comparison of anxiety, depression and sexual life satisfaction in two groups of fertile women in Tehran. J Reprod Infertil. 2011;12(2):157-162.

22. Mohammadi Hosseini F, Farzadi L, Seyed Fatemi N. Tense factors and coping strategies in infertile women referring to Alzahra Training Clinical Center of Tabriz (1998). J Reprod Infertil. 2001;2(3):27-34.

23. Latifnejad Roudsari R, Karami DA, Esmaili HA, Mousavifar N, Agha MS. The relationship between body image and marital adjustment in infertile women. Iranian J Obstet Gynecol Infertil. 2011;14(6):9-19.

Copyright (C) 2017 The Author (s); This is an open-access article distributed under the terms of the Creative Commons Attribution License (http://creativecommons.org/licenses/by/4.0), which permits unrestricted use, distribution, and reproduction in any medium, provided the original work is properly cited. 